\title{
Transient Aeroelastic Responses of Folding Wing in Morphing Motion
}

\author{
Yingge $\mathrm{Ni}^{1}$, Chi Hou ${ }^{1}$, Xiaopeng $\mathrm{Wan}^{1}$ \& Meiying Zhao ${ }^{1}$ \\ ${ }^{1}$ School of Aeronautics, Northwestern Polytechnical University, Xi'an 710072, China
}

Keywords: folding wing, time-varying system, rational function approximation, double lattice method.

\begin{abstract}
To investigate the transient aeroelastic responses of a folding wing during morphing motion, a time-varying aeroelastic equation in state space is presented. The structural model is established based on the component modal synthesis method. A time-dependent transformation is introduced to establish the compatibility equations. The aerodynamic force is obtained by double lattice method, and then the rational function fitting method is adopted to deduce the approximate expressions of the aerodynamic force in time domain which is used in conjunction with the structural model. The equations of motion in state space are solved using the Runge-Kutta numerical integration technique to predict the transient aeroelastic responses. The effects of the flow velocity and the morphing velocity are studied to deeply understand the aeroelastic characteristics of a folding wing. The numerical results indicate that the aerodynamic force contributes to the morphing motion. With a greater flow velocity or a slower morphing motion, the reaction moment will decrease. So a better aeroelastic performance can been obtained.
\end{abstract}

\section{Introduction}

The morphing aircraft is to achieve a broader range of operational mode, which can change its shape and size to adapt to dynamic mission environments. The morphing aircraft is drawing aircraft designers' attention. So the DARPA' MAS program are examples of larger coordinated efforts to develop morphing aircraft. One strong candidate for morphing aircraft design is a folding wing configuration [1-4]. However, the rapid changes in the wing shape and size will raise difficult aeroelastic problems regarding the time-dependent aerodynamics and the structure during the largescale morphing motion. So transient aeroelastic responses become very important during the morphing motion.

Several approaches have been suggested for morphing aeroelastic problems in previous work. Reich et al [5] and Scarlett et al [6] proposed an aeroelastic simulation of a folding wing through integration of finite element modelling, continuous-time multibody dynamics, aerodynamic estimation and flight control. Liu et al [7] presented a continuous dynamic simulation of nonlinear aerodynamic-nonlinear structures interactions (NANSI) methodology for morphing wing aeroelasticity, which combined the structural equations of motion in generalized modal coordinates and computational fluid dynamics solution. Selitrennik et al [8] presented an approach for computational fluid dynamics-based aeroelastic simulation of morphing flight vehicles, in which the fictitious-mass modal synthesis and time-varying coordinate transformation matrix were introduced to establish the aerodynamic motion equation. Zhao et al [9] developed a set of differentialalgebraic equations that governed the time evolution of the folding wing during the morphing process by using the floating frame approach, in which the CFD code was not taken into account.

In the present study, a first-order, state-space model for transient aeroelastic analysis of a folding wing during morphing motion is developed based on component modal synthesis method and rational function approximation to aerodynamic force in time domain which is obtained by doublet lattice method (DLM). The Runge-Kutta numerical integration technique is adopted to predict the transient aeroelastic responses. The effects of the aerodynamic force, the flow velocity and the morphing velocity on the aeroelastic performance are investigated and provide a deeper understanding of folding wing transient behaviour. 


\section{Equation of motion}

A geometric model of a folding wing is shown in Fig.1. The folding wing model is separated into three components: component $\mathrm{A}$, namely the fuselage; component $\mathrm{B}$ is the inboard wing; and component $\mathrm{C}$ is the outboard wing. They are attached through a hinge that is modelled as a set of torsional springs at several points. Each torsional spring stiffness is $K_{A}$ and $K_{B}$, respectively. The folding angles between component $\mathrm{A}$ and $\mathrm{B}$ and component $\mathrm{B}$ and $\mathrm{C}$ are $\theta_{\mathrm{B}}$ and $\theta_{C}$. Here, component $\mathrm{A}$ is assumed to be a clamped-free plate, and the component $\mathrm{B}$ and $\mathrm{C}$ are assumed to be free-free plates.

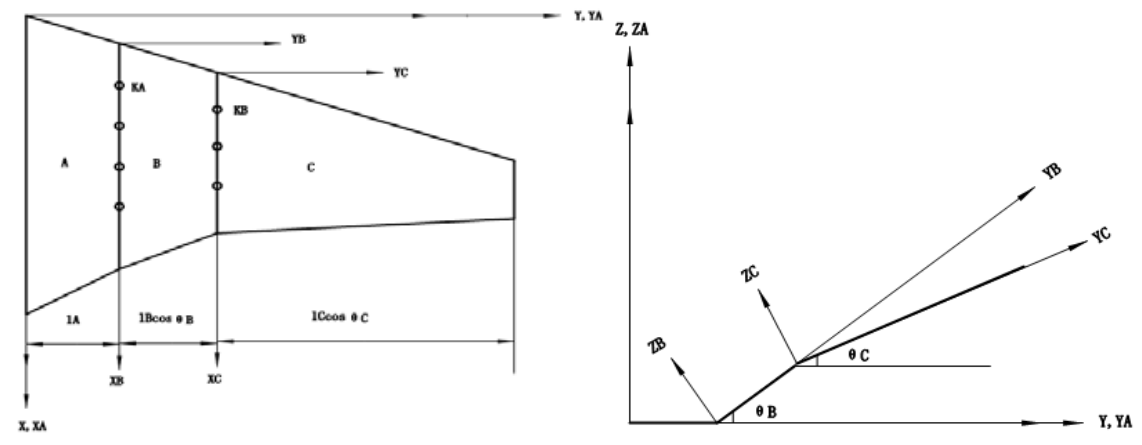

Fig.1: Folding wing geometry and coordinate frames

Structural dynamic equations. The dynamic equation of each component can be written as follows in physical space:

$$
\mathbf{M}_{i} \ddot{\mathbf{u}}_{i}+\mathbf{C}_{i} \dot{\mathbf{u}}_{i}+\mathbf{K}_{i} \mathbf{u}_{i}=\mathbf{F}_{a}^{i}+\mathbf{F}_{g}^{i}+\mathbf{F}_{c}^{i},(i=A, B, C)
$$

where $\mathbf{M}_{i}, \mathbf{C}_{i}$ and $\mathbf{K}_{i}$ are the mass, damping and stiffness matrices of eachcomponent, respectively. $\mathbf{u}_{i}$ is the physical coordinate. $\mathbf{F}_{a}^{i}, \mathbf{F}_{g}^{i}$ and $\mathbf{F}_{c}^{i}$ represent the aerodynamic forces, the gravity and the constraint forces of the attached points.

As for $\mathbf{F}_{c}^{i}$ of the attached points of components A and B is taken as an example,

$$
\begin{aligned}
& \left.\quad \mathbf{m}_{\theta}\right|_{y A=I A}=K_{A}\left(\left.\mathbf{u}_{\theta}\right|_{y A=I A}-\left.\mathbf{u}_{\theta}\right|_{y B=0}\right),\left.\quad \mathbf{m}_{\theta}\right|_{y B=0}=-K_{A}\left(\left.\mathbf{u}_{\theta}\right|_{y A=I A}-\left.\mathbf{u}_{\theta}\right|_{y B=0}\right) \\
& \text { So }\left.\mathbf{m}_{\theta}\right|_{y A=I A}+\left.\mathbf{m}_{\theta}\right|_{y B=I 0}=\mathbf{0} .
\end{aligned}
$$

Similarly, the deduction process holds true for the attached points of components B and C. For the whole folding wing, the dynamic equation can be written

$$
\mathbf{M u ̈}+\mathbf{C} \dot{\mathbf{u}}+\mathbf{K u}=\mathbf{F}_{a}+\mathbf{F}_{g}
$$

The coupling between components A and B and components B and C is introduced by enforcing displacement compatibility at the interfaces.

$$
\mathbf{u}=\mathbf{T}\left(\theta_{B}, \theta_{C}\right) \mathbf{u}_{1}
$$

where $\mathbf{T}$ is a transformation matrix, and $\mathbf{u}_{1}$ is a displacement vector.

The differentiation of the equation above with respect to time yields

$$
\dot{\mathbf{u}}=\mathbf{T} \dot{\mathbf{u}}_{1}+\dot{\mathbf{T}} \mathbf{u}_{1}, \quad \ddot{\mathbf{u}}=\mathbf{T} \ddot{\mathbf{u}}_{1}+2 \dot{\mathbf{T}} \dot{\mathbf{u}}_{1}+\ddot{\mathbf{T}} \mathbf{u}_{1}
$$

The substitution of Eqs.(5) and (6) into Eq.(4), and the premultiplication by $\mathbf{T}^{T}$ yield the nonlinear equation of motion.

$$
\mathbf{M}_{1} \ddot{\mathbf{u}}_{1}+\mathbf{C}_{1} \dot{\mathbf{u}}_{1}+\mathbf{K}_{1} \mathbf{u}_{1}=\mathbf{T}^{\mathrm{T}} \mathbf{F}_{a}+\mathbf{T}^{\mathrm{T}} \mathbf{F}_{g}
$$

The generalized matrices on the left side of Eq.(7) are

$$
\mathbf{M}_{1}=\mathbf{T}^{\mathrm{T}} \mathbf{M T}, \mathbf{C}_{1}=\mathbf{T}^{\mathrm{T}} \mathbf{C} \mathbf{T}+2 \mathbf{T}^{\mathrm{T}} \mathbf{M} \dot{\mathbf{T}}, \mathbf{K}_{1}=\mathbf{T}^{\mathrm{T}} \mathbf{K} \mathbf{T}+\mathbf{T}^{\mathrm{T}} \mathbf{C} \dot{\mathbf{T}}+\mathbf{T}^{\mathrm{T}} \mathbf{M} \ddot{\mathbf{T}}
$$

Generally, aeroelastic analysis is conducted in the modal coordinate to reduce the computation time. By introducing the equation $\mathbf{u}_{1}=\boldsymbol{\varphi q}$, the dynamic equation can be written in the modal space. 


$$
\begin{gathered}
\mathbf{M}_{m} \ddot{\mathbf{q}}+\mathbf{C}_{m} \dot{\mathbf{q}}+\mathbf{K}_{m} \mathbf{q}=\mathbf{F}_{m a}+\mathbf{F}_{m g} \\
\text { where } \mathbf{M}_{m}=\varphi^{\mathrm{T}} \mathbf{M}_{1} \boldsymbol{\varphi}, \mathrm{C}_{m}=\varphi^{\mathrm{T}} \mathrm{C}_{1} \boldsymbol{\varphi}, \mathbf{K}_{m}=\varphi^{\mathrm{T}} \mathbf{K}_{1} \boldsymbol{\varphi}, \mathrm{F}_{m a}=\varphi^{\mathrm{T}} \mathbf{T}^{\mathrm{T}} \mathrm{F}_{a}, \mathrm{~F}_{m g}=\varphi^{\mathrm{T}} \mathrm{T}^{\mathrm{T}} \mathrm{F}_{g} .
\end{gathered}
$$

Rational function approximation of aerodynamic model. The most generally used scheme is DLM which solves acceleration potential equations in the frequency domain. The detail computation of DLM can be found in Ref. [10]. The relationship between the aerodynamic force acting on the nodal point and the vertical displacement of the nodal point is established by the infinite plate splining method. Then, the generalized aerodynamic force can be written as

$$
\mathbf{F}_{m a}=q_{d} \mathbf{Q}\left(M_{\infty}, k\right) \mathbf{q}
$$

where $q_{d}$ is the dynamic pressure, $\mathbf{Q}\left(M_{\infty}, k\right)$ is the generalized aerodynamic matrix, which can be obtained for a series of reduced frequency $k$ values and a given Mach number $M_{\infty}$.

As for the aeroelastic model in the state space, the generalized aerodynamic in frequency domain is not accessible. Thus, the aerodynamic matrix should be approximated as a rational function [11]. At present, there are several methods of rational function approximation. Here, the minimal state method is adopted. The approximation form is as follows:

$$
\mathbf{Q}(\overline{\mathrm{s}})=\mathbf{A}_{0}+\mathbf{A}_{1} \overline{\mathrm{s}}+\mathbf{A}_{2} \overline{\mathrm{s}}^{2}+\mathbf{D}_{s}\left(\mathbf{I} \overline{\mathrm{s}}-\mathbf{R}_{\mathrm{s}}\right)^{-1} \mathbf{E}_{\mathrm{s}} \overline{\mathrm{s}}
$$

where $\mathbf{A}_{0}, \mathbf{A}_{1}, \mathbf{A}_{2}, \mathbf{D}_{s}$ and $\mathbf{E}_{s}$ are unknown matrices, which can be obtained least square fit. $\mathbf{I}$ is an identity matrix, $\bar{s}=s b / V, s$ is Laplace variable, $b$ is the reference length, $V$ is the flow velocity .

\section{State space equation.}

The Eq.(9) becomes

$$
\mathbf{M}_{m} \ddot{\mathbf{q}}+\mathbf{C}_{m} \dot{\mathbf{q}}+\mathbf{K}_{m} \mathbf{q}=q_{d}\left(\mathbf{A}_{0}+\mathbf{A}_{1} \overline{\mathbf{s}}+\mathbf{A}_{2} \overline{\mathbf{s}}^{2}\right) \mathbf{q}+q_{d} \mathbf{D}_{s}\left(\overline{\mathbf{I}}-\mathbf{R}_{s}\right)^{-1} \mathbf{E}_{s} \bar{s} \mathbf{q}+\mathbf{F}_{m g}
$$

For convenience, the equation above can be written in a more compact form as

$$
\begin{gathered}
\overline{\mathbf{M}} \ddot{\mathbf{q}}+\overline{\mathbf{C}} \dot{\mathbf{q}}+\overline{\mathbf{K}} \mathbf{q}=q_{d} \mathbf{D}_{s}\left(\mathbf{I} \bar{s}-\mathbf{R}_{s}\right)^{-1} \mathbf{E}_{s} \overline{\mathbf{s}} \mathbf{q}+\mathbf{F}_{m g} \\
\text { where } \overline{\mathbf{M}}=\mathbf{M}_{m}-q_{d}(b / V)^{2} \mathbf{A}_{2}, \overline{\mathbf{C}}=\mathbf{C}_{m}-q_{d}(b / V) \mathbf{A}_{1}, \overline{\mathbf{K}}=\mathbf{K}_{m}-q_{d} \mathbf{A}_{0}
\end{gathered}
$$

Introducing $\mathbf{q}_{a}=\left(\mathbf{I} \bar{s}-\mathbf{R}_{s}\right)^{-1} \mathbf{E} \bar{s} \overline{\mathbf{q}}$, which $\mathbf{q}_{a}$ satisfies $\dot{\mathbf{q}}_{a}=\mathbf{R}_{s}(V / b) \mathbf{q}_{a}+\mathbf{E}_{s} \dot{\mathbf{q}}$, so the Eq.(15) can be converted to the time-variant state space form as follows:

$$
\left\{\begin{array}{c}
\dot{\mathbf{q}} \\
\ddot{\mathbf{q}} \\
\dot{\mathbf{q}}_{a}
\end{array}\right\}=\left[\begin{array}{ccc}
\mathbf{0} & \mathbf{I} & \mathbf{0} \\
-\overline{\mathbf{M}}^{-1} \overline{\mathbf{K}} & -\overline{\mathbf{M}}^{-1} \overline{\mathbf{C}} & q_{d} \overline{\mathbf{M}}^{-1} \mathbf{D}_{s} \\
\mathbf{0} & \mathbf{E}_{s} & (V / b) \mathbf{R}_{s}
\end{array}\right]\left\{\begin{array}{c}
\mathbf{q} \\
\dot{\mathbf{q}} \\
\mathbf{q}_{a}
\end{array}\right\}+\left\{\begin{array}{c}
\mathbf{0} \\
\mathbf{F}_{m g} \\
\mathbf{0}
\end{array}\right\}
$$

The equation above can be solved using time-marching scheme such as the Runge-Kutta [12].

\section{Results and discussion}

The components A, B and C are made of aluminium plate modelled by CQUAD4 element with a 2mm thickness in MSC.NASTRAN. The attachment points of each component are equal interval in Fig.2. At each attachment point, the torsional stiffness is equal. To predict the transient responses, the fold angles satisfy the following equations

$$
\begin{aligned}
& \theta_{B}(t)=\left\{\begin{array}{l}
\frac{120}{t_{s}} \times t,\left(0 \leq t \leq t_{s}\right) \\
120 \mathrm{deg},\left(t>t_{s}\right)
\end{array}\right. \\
& \theta_{C}(t)=0
\end{aligned}
$$

where 120 deg means the completion of morphing motion in $t_{s}$ seconds. When the aerodynamic force is taken into account, the results were obtained in the case of incompressible flow $\left(M_{\infty}=0\right)$ and $K_{A}=K_{B}=1000 \mathrm{Nmm} / \mathrm{rad}$. 


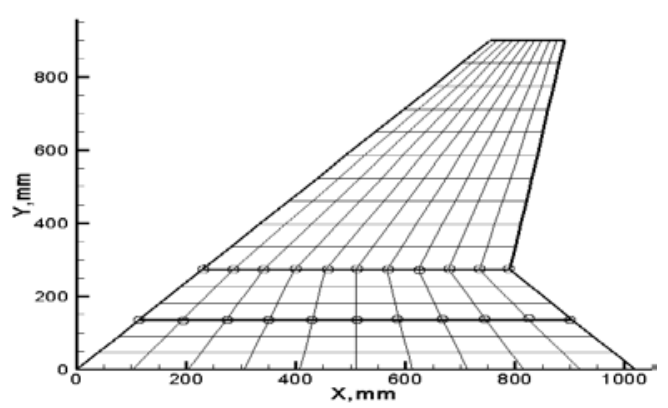

Fig.2: Finite element model of folding wing

Transient responses under gravity. In the beginning, the aerodynamic force is neglected, namely, there are no items about aerodynamics in Eq.(16). The tip vertical displacement and the reaction moment are shown in Fig. 3 and Fig. 4. For convenience, the reaction moment between components $\mathrm{A}$ and $\mathrm{B}$ is named inner reaction moment while the reaction moment between components $\mathrm{B}$ and $\mathrm{C}$ is named outer reaction moment. There is a great oscillation in both the tip vertical displacement and the reaction moment during the morphing motion. As time increase, the amplitudes of oscillation decay and approach to a stable value. The stable value of the displacement is $100.86 \mathrm{~mm}$. The reaction moments are $689 \mathrm{Nmm}$ and $5.408 \mathrm{Nmm}$, respectively. As for the reaction moment, the outer reaction moment is much greater than the inner reaction moment. The moment at outer hinge due to the gravity of the inner wing and outer wing superimposed while the moment at inner hinge due to the inner and outer wing's gravity weakened. So there is a great difference between the outer reaction and inner reaction. The final reaction moments are reasonable.

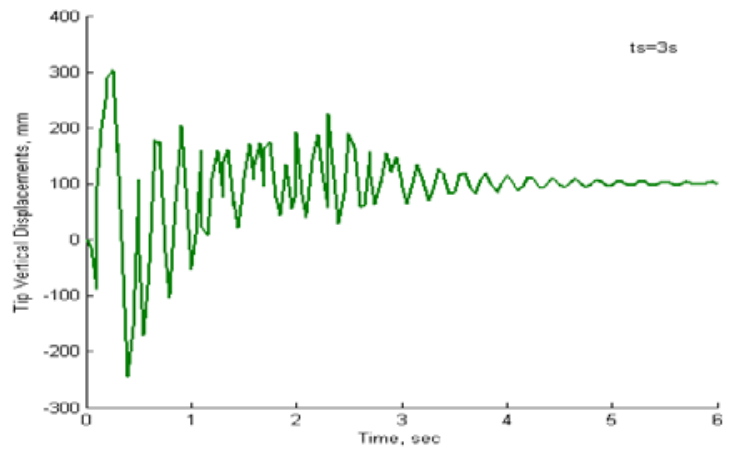

Fig.3: Tip vertical displacement in absence of aerodynamic force

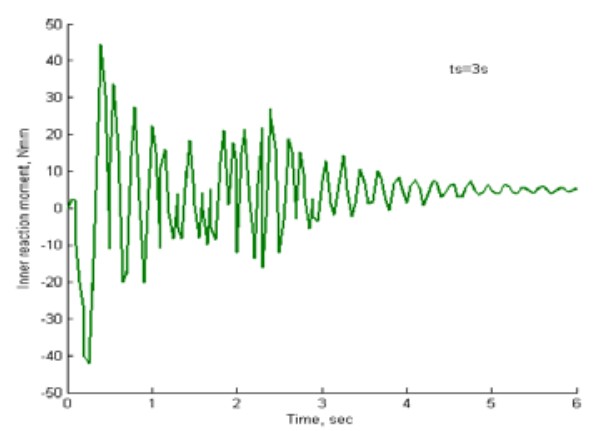

(a) Inner reaction moment

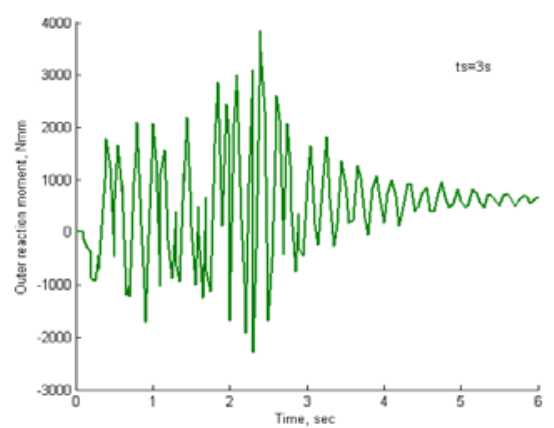

(b) Outer reaction moment

Fig. 4: Reaction moment in absence of aerodynamic force

Transient responses under gravity and aerodynamic force. In the following simulations, the aerodynamic force is taken into account in Eq.(16). The transient responses at a different flow velocity with $t_{s}=3 \mathrm{~s}$ are shown in Fig. 5 . As expected, when the morphing motion is completed, the tip vertical displacement nearly reaches $118.6 \mathrm{~mm}$. During the morphing motion, the displacement has oscillations whatever the velocity. The amplitudes of oscillation decay while the morphing motion is finished. Compared with the Fig.3, the oscillation of tip vertical displacement is much smaller. With the aerodynamic, the morphing motion became gentle. In addition, the displacement nearly stabilizes after morphing motion. Nearly at $2.25 \mathrm{~s}$, the displacement reaches the maximum 
value. Now, the inner wing is perpendicular to the fuselage and the outer wing.

In Fig.6, as the time increases, the reaction moments tend to stable values, i.e. $498.3 \mathrm{Nmm}$ at $V=20 \mathrm{~m} / \mathrm{s}, 458.6 \mathrm{Nmm}$ at $V=25 \mathrm{~m} / \mathrm{s}, 421.2 \mathrm{Nmm}$ at $V=30 \mathrm{~m} / \mathrm{s}$ in the outer reaction moment; $3.916 \mathrm{Nmm}$ at $V=20 \mathrm{~m} / \mathrm{s}, 3.607 \mathrm{Nmm}$ at $V=25 \mathrm{~m} / \mathrm{s}, 3.316 \mathrm{Nmm}$ at $V=30 \mathrm{~m} / \mathrm{s}$ in the inner reaction moment. With an increase in the flow velocity, the stable values of the reaction moment decrease. Because the aerodynamics is normal to the wing and upward. So it plays a positive role in the morphing motion. But the outer reaction moment still exceeds the inner reaction moment. With these velocities, the moment due to aerodynamic force is smaller than one due the gravity. The inner reaction moment just fluctuates while the outer reaction moment first rises, and then stabilizes. In this study, it is worth of noticing that the increase in the flow velocity would be an efficient approach for the decrease in the reaction moment.

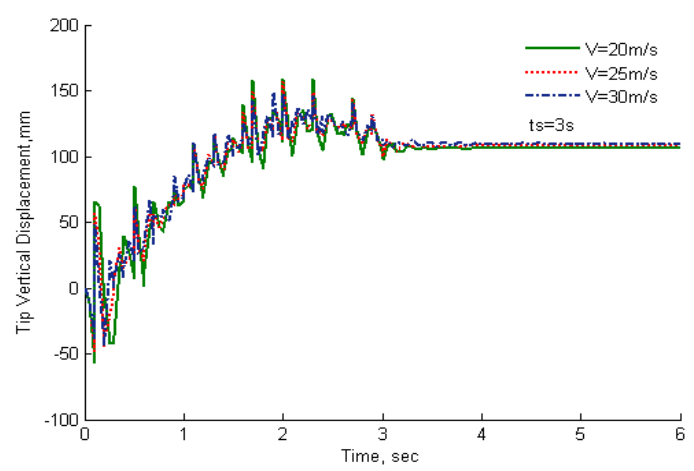

Fig. 5: Tip vertical displacement at several flow velocities

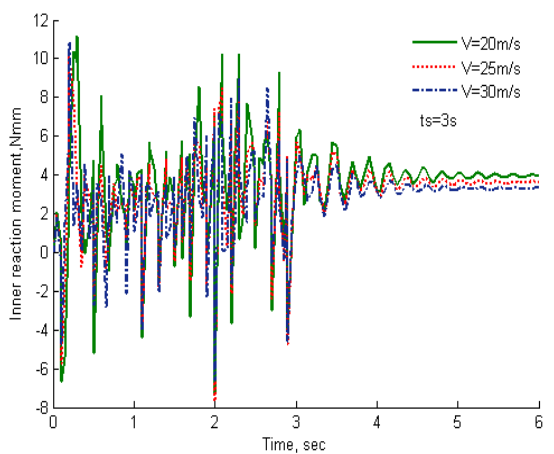

(a) Inner reaction moment

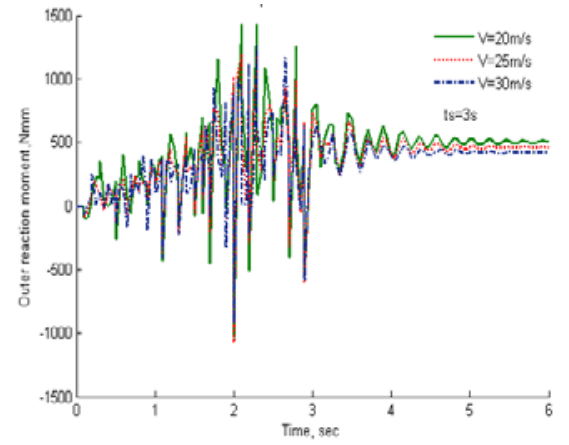

(b) Outer reaction moment

Fig. 6: Reaction moment at several flow velocities

Considering the influence of the morphing speed, the tip vertical displacement and reaction moment are calculated during the morphing motion at $V=25 \mathrm{~m} / \mathrm{s}$. In Fig.7 and Fig.8, the tip vertical displacements nearly approach to the same value when the motion is completed. The displacement peaks at $2.25 \mathrm{~s}, 1.5 \mathrm{~s}$ and $1.13 \mathrm{~s}$, respectively when the inner wing is perpendicular to the fuselage and the outer wing. So the displacement curve is right in Fig.7.

However, there is a slight difference in stable values of the reaction moment, i.e. $458.6 \mathrm{Nmm}$ at $t_{s}=3 \mathrm{~s}, 479 \mathrm{Nmm}$ at $t_{\mathrm{s}}=2 \mathrm{~s}, 494.9 \mathrm{Nmm}$ at $t_{\mathrm{s}}=1.5 \mathrm{~s}$ in the outer reaction moment; $3.607 \mathrm{Nmm}$ at $t_{s}=3 \mathrm{~s}, 3.707 \mathrm{Nmm}$ at $t_{s}=2 \mathrm{~s}, 3.774 \mathrm{Nmm}$ at $t_{s}=1.5 \mathrm{~s}$ in the inner one. So with an increase in the morphing velocity, the stable value of the reaction moment has a slight increase. It is also noted that the decrease in the morphing velocity can contribute to the decrease in the stable value of reaction moments. 


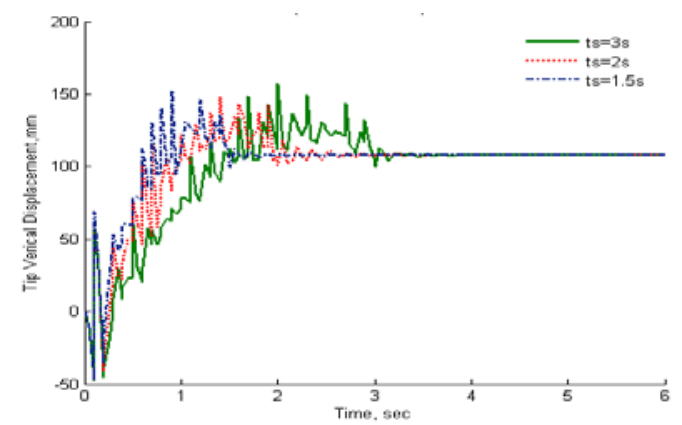

Fig.7: Tip vertical displacement at several morphing velocities

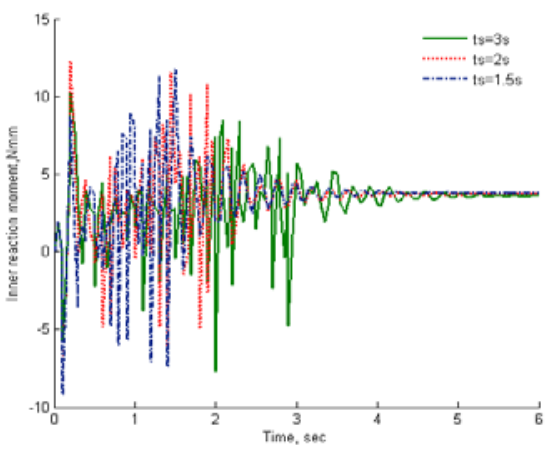

(a) Inner reaction moment

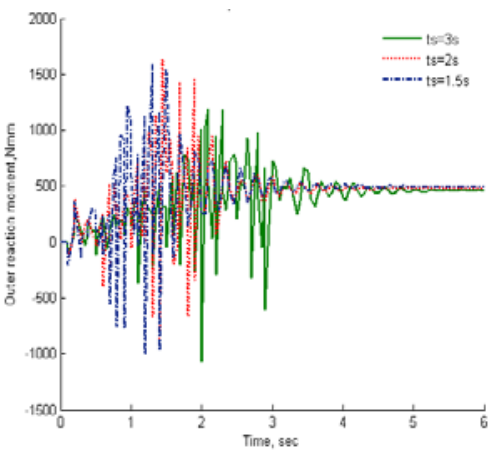

(b) Outer reaction moment

Fig. 8: Reaction moment at several morphing velocities

It is noted that when the aerodynamic is taken into account, there is a decrease in the reaction moments. And the fluctuation is smaller. So the aerodynamic force plays a positive role in the morphing motion. The influence of the morphing velocity and flow velocity on the transient responses of folding wing is not significant.

\section{Conclusions}

Based on the component modal synthesis method and rational function approximation to aerodynamic force, a time-variant state space equation for the morphing motion of a folding wing is presented in this paper. The inclusion of the time-dependent transformation brings some nonlinear terms into the equation of motion. The established equation can be used to obtain the transient aeroelastic responses during the morphing motion. The results show that the aerodynamic force can contribute to the morphing motion. The reaction moment is not greatly sensitive to the flow velocity and morphing velocity. However, an increase in flow velocity leads to a decrease in the reaction moment while a decrease in morphing velocity results in a decrease in the reaction moment.

\section{Acknowledgements}

Corresponding author: Xiaopeng Wan

E-mail address:wanxp@nwpu.edu.cn

\section{References}

[1] J. R. Wilson. Morphing UAVs Change the shape of sarfare, Aerospace America, 42, pp.28-29, 2004.

[2] D.H Lee\&Terrence A. Weisshaar. Aeroelastic studies on a folding wing configuration. AIAA/ASME/ASCE/AHS/ASC Structural Dynamics and Materials Conference, Texas, AIAA 20051996, April 2005.

[3] B. Sanders, F. E. Eastep\&E. Forster. Aerodynamic and aeroelastic charateristics of wings with 
conformal control surfaces for morphing aircraft. Journal of Aircraft, 40(1),pp.94-99,2003.

[4] Deman Tang \& Earl H. Dowell. Theoretical and experimental aeroelastic study for folding wing strcutures. Journal of Aircraft, 45(4),pp.1136-1147, 2008.

[5] Gregory W. Reich, Jason C.Bowman \& Brian Sanders. Development of an integrated aeroelastic multibody morphing simulation tool. 47th AIAA/ASME/ASCE/AHS/ASC Structures, Structural Dynamics, and Materials Conference, Rhode Island, AIAA 2006-1892,May 2006.

[6] John N.Scarlett, Robert A. Canfield \& Brian Sanders. Multibody dynamic aeroelastic simulation of a folding wing aircraft. 47th AIAA/ASME/ASCE/AHS/ASC Structures, Structural Dynamics, and Materials Conference. Rhode Island, AIAA 2006-2153,May 2006.

[7] Liu D.D, Chen P.C, Zhang. Z, Wang Z, Yang S. Lee. D.H. et al. Continuous dynamic simulation for morphing wing aeroelasticity. 50th AIAA/ASME/ASCE/AHS/ASC Structures, Structural Dynamics, and Materials Conference,California, AIAA 2009-2572, May 2009.

[8] Evgeny Selitrennik, Moti Karpel \&Yuval Levy. Computational aeroelastic simulation of rapidly morphing air vehicles.Journal of Aircraft, 49(6),pp.1675-1686, 2012.

[9] Zhao Y.H.\&Hu H.Y. Prediction of transient responses of a folding wing during the morphing process.Aerospace Science and Technology,24, pp.89-94,2013.

[10] Guan De. Unsteady aerodynamic calculation.Beihang Press 1991.

[11] Zhao Yong-hui. Aeroelastic and Control. Science Press 2007.

[12] Won Young Yang, Wenwu Cao \& Tae-Sang Chung, John Morris,Applied Numerical Methods Using Matlab. John Wiley \& Sons, 2005. 